\title{
Uso de la metodología FMECA -RCM, para la optimización De la estrategia de mantenimiento en una planta de tostación de cobre
}

\section{Using FMECA - RCM methodology as a tool for the optimization of the maintenance strategy in a copper roasting plant}

\section{Edgar Estupiñan ${ }^{1}$ y Oscar Cordero $^{2}$}

${ }^{1}$. Departamento de Ingeniería Mecánica, Universidad de Tarapacá (Arica, Chile). eestupin@uta.cl

${ }^{2}$ Ingeniero de Mantenimiento, Codelco, División Ministro Hales. oscar.cordero.arenas@ gmail.com

\section{Resumen}

Los cambios y variaciones cada vez más frecuentes en los precios del cobre, dejan de manifiesto, la necesidad de que las empresas mineras sean cada vez más rigurosas en el control de costos de producción, manteniendo su competitividad en el mercado internacional. De esta manera, el uso e implementación de nuevas estrategias de mantenimiento están teniendo una mayor acogida, dado que pueden permitir contener y bajar los costos asociados a la producción. En este trabajo, se presenta el caso de una empresa minera de Chile, la cual se ha propuesto reducir significativamente sus costos de producción, lo que implica necesariamente dirigir esfuerzos en aquellos procesos que regularmente presentan baja disponibilidad y altos costos de mantenimiento, como lo es en este caso, el proceso de tostación. Por lo tanto, mediante un análisis de sensibilidad, se demuestra la conveniencia de implementar un plan de mantenimiento basado en la confiabilidad, utilizando la metodología FMECA, que con el apoyo de herramientas matemáticas y estadísticas, permite tener información relacionada con el estado de los equipos, así como también, permite identificar brechas y oportunidades de mejora de manera priorizada.

Palabras clave: Confiabilidad, FMECA, Gestión de
Mantenimiento, Minería.

\section{Abstract}

The changes and variations in copper price, each time more often, has shown the challenge of being more rigorous in the control of production costs in the mining industry. Therefore, the use and implementation of new maintenance strategies should be adopted in order to control and lower the costs associated to the production costs. The case of a mining company in Chile, which has priorized as a goal, to reduce production costs significantly, is presented in this work. Thus, the main efforts were concentrated on those processes with lower availability and higher maintenance costs, such as, it is the case of the roasting process. Therefore, by means of a sensitivity analysis, it is showed the convenience of implementing a reliability centered maintenance plan, by using the FMECA methodology, with the support of mathematical and statistic tools, that allow to get information about the condition of machines, as well as, to identify gaps and opportunities for improvement.

Keywords: Reliability, FMECA, Maintenance Management, Mining. 
197

\section{Introducción}

Las fluctuaciones de precios de los minerales son cada vez más frecuentes, dados los constantes cambios en los contextos geopolíticos y económicos a nivel global, como también de los factores climáticos, entre otros aspectos, que van más allá del ámbito industrial. De esta manera, las compañías mineras requieren mantener en niveles óptimos los costos de producción, y por tanto deben consecuentemente disminuir los gastos asociados a mantenimiento. El presente estudio se centra en una planta de tostación de cobre de una importante compañía minera de cobre en Chile, en donde sus directivos se han propuesto alcanzar un nivel de disponibilidad para los equipos de la planta del 95\%, como también, disminuir los costos asociados al mantenimiento, los cuales superan actualmente a lo planificado. El equipo de mayor criticidad que debido a sus detenciones imprevistas, afecta la disponibilidad de la planta de Tostación, corresponde al horno de tostación. Por lo tanto, mediante un estudio de sensibilidad utilizando la metodología FMECA-RCM ${ }^{1}$, se busca proponer mejoras que permitan optimizar el plan de gestión de mantenimiento, y consecuentemente mejorar los indicadores de confiabilidad y disponibilidad de los equipos críticos de la planta de tostación.

\section{Metodología}

La metodología FMECA (Luthra, 1991) permite analizar la funcionalidad de los equipos a partir de sus modos de falla, estableciendo su jerarquía, lo que contribuye a poder elaborar planes de mantenimiento focalizados en aquellas fallas de mayor criticidad de los equipos. $\mathrm{La}$ implementación del FMECA consta de cinco etapas principales:
Contexto del equipo. Se deben identificar los modos de operación de los equipos analizados, como también las necesidades desde el punto de vista del usuario/operario. También se deben identificar todos los parámetros operacionales.

Análisis Funcional. Se deben identificar las fallas que provocan pérdida de función o funciones de los equipos analizados.

Identificación de los modos de fallas. Se identifica la forma en que el equipo pierde su función, cada modo de falla corresponderá a una tarea de mantenimiento.

Efectos y consecuencias de una falla. En esta etapa se considera como falla se manifiestan las fallas y sus consecuencias (de producción, personales o ambientales).

Jerarquización del Riesgo. La jerarquización permite optimizar las acciones y recursos de mantenimiento (ISO, 2011). El Número Probabilístico de Riesgo; NPR = D.O.S; dónde; D: Detectabilidad; O: Ocurrencia; S: Severidad.

Una vez realizada la priorización, se aplica la metodología RCM, especialmente a aquellos modos de falla de mayor ponderación (Moubray, 2001).

Para la estimación de las tasas de fallas y el nivel de confiabilidad, se utiliza la distribución de Weibull (Quintana, et al., 2015), cuya función general está definida por la ecuación 1.

$f(t)=\frac{\beta}{\eta} \times\left[\frac{(t-\gamma)}{\eta}\right]^{\beta-1} \times e^{-((t-\gamma) / \eta)^{\beta}}$

Dónde:

$f(t) \geq 0, \quad t \geq \gamma, \beta>0, \eta>0,-\infty<\gamma<\infty, \eta$ $=$ parámetro de escala; $\beta$ = parámetro de forma;

${ }^{1}$ FMECA: Failure Mode Effect Cause Analysis; RCM: Reliability Centered Maintenance 
198

$\gamma=$ parámetro de ubicación.

De la función de distribución acumulada de fallas se obtiene:

- Función de Confiabilidad.

$$
R(t)=1-f(t)
$$

- Distribución de Falla

$$
\lambda(t)=\frac{\beta}{\eta}\left(\frac{t}{\eta}\right)^{\beta-1}
$$

\section{Resultados Obtenidos}

Las detenciones del complejo, son registradas por el sistema de eventos planta. Para el presente estudio se utilizan datos registrados en el sistema, durante el periodo comprendido entre julio 2015 y julio 2016, relacionados con las detenciones imprevistas asignadas a mantenimiento mecánico, de los equipos críticos, que corresponden a aquellos equipos que por causa de un fallo inesperado, detienen el proceso de tostación.

\subsection{Determinación de equipos críticos}

El complejo de Tostación, es la planta con la disponibilidad más baja dentro de la Compañía minera, correspondiendo a un $85 \%$. Al elaborar un diagrama de Pareto para determinar los equipos que más impactan en las detenciones del complejo de tostación, se identifican los equipos más críticos; seis de los cuales concentran el $95 \%$ de las detenciones del complejo de Tostación, como se muestra en la tabla 1.

Tabla 1. Equipos de mayor criticidad

\begin{tabular}{|l|c|c|}
\hline Equipo & $\begin{array}{c}\text { Horas de } \\
\text { detención }\end{array}$ & $\begin{array}{c}\% \\
\text { Acumulado }\end{array}$ \\
\hline Enfriador 1 & 239,451 & $55,0 \%$ \\
\hline Rastra 3 & 81,71 & $73,8 \%$ \\
\hline Tornillo 14 & 41,26 & $83,3 \%$ \\
\hline Enfriador 2 & 29,448 & $90,0 \%$ \\
\hline $\begin{array}{l}\text { Transporte } \\
\text { Neumático }\end{array}$ & 13,55 & $93,1 \%$ \\
\hline
\end{tabular}

\begin{tabular}{|l|c|c|}
\hline Tornillo 13 & 8,4 & $95,1 \%$ \\
\hline
\end{tabular}

\subsection{Análisis de confiabilidad de equipos críticos}

Para determinar el nivel de confiabilidad de los equipos principales se utiliza la distribución de Weibull de 2 parámetros (Waghmode y Sahasrabudhe, 2012), siendo este, el modelo de distribución de probabilidad que mejor se adapta a equipos electromecánicos. En la tabla 2, se muestra el resumen de los resultados obtenidos a partir de las curvas de densidad de falla $y$ probabilidad obtenidas para cada uno de los equipos críticos (Cordero y Estupiñan, 2018).

Tabla 2. Parámetros de Confiabilidad, equipos críticos planta de tostación

\begin{tabular}{|l|c|c|c|c|c|}
\hline Equipo & MTTR & MTBF & $\boldsymbol{\beta}$ & $\boldsymbol{\eta}$ & B50 \\
\hline Enfriador 1 & 6.8 & 250.2 & 0.67 & 189.9 & 110.1 \\
\hline Rastra 3 & 3.1 & 574.1 & 0.54 & 323.5 & 163.2 \\
\hline Tornillo 14 & 5.9 & 461.1 & 1.96 & 520 & 431.3 \\
\hline Enfriador 2 & 4.2 & 255.7 & 1.77 & 287.3 & 233.6 \\
\hline $\begin{array}{l}\text { Transporte } \\
\text { Neumático }\end{array}$ & 2.3 & 674.2 & 6.5 & 723.3 & 683.9 \\
\hline Tornillo 13 & 1.7 & 237.4 & 1.46 & 262 & 203.7 \\
\hline
\end{tabular}

MTTR: Mean time to repair; MTBF: Mean time between failure.

De los resultados obtenidos se puede destacar lo siguiente:

- Para el enfriador 1 y la rastra 3, la densidad de fallas tiene una forma decreciente, dado el factor $\beta<1(\beta=0,67 ; \beta=0,54)$. Por tanto, se puede deducir que ambos equipos están en una etapa de fallas prematuras.

- Para el tornillo 14, enfriador 2, transporte neumático y tornillo 13 , el factor $\beta>1$, indica que las tasas de falla son crecientes. Por tanto, se puede deducir que estos equipos están en una etapa de fallas por vejez. 
199

- El enfriador 1 es el equipo con mayor número de horas de detención, por tanto con menor disponibilidad y mayor criticidad. El tiempo medio entre fallas es de 250,2 horas, con un $30 \%$ de probabilidad. El factor $\eta=189,9$, implica que sobre este número de horas se concentra el $63,2 \%$ de las fallas. La probabilidad de falla de un $50 \%$ está en las 110 horas, esto se denota por el valor de B50. Las curvas de densidad de falla y probabilidad para el enfriador 1 , se muestran en la tabla 3.

Tabla 3. Enfriador 1- Densidad de falla, confiabilidad y parámetros de Weibull

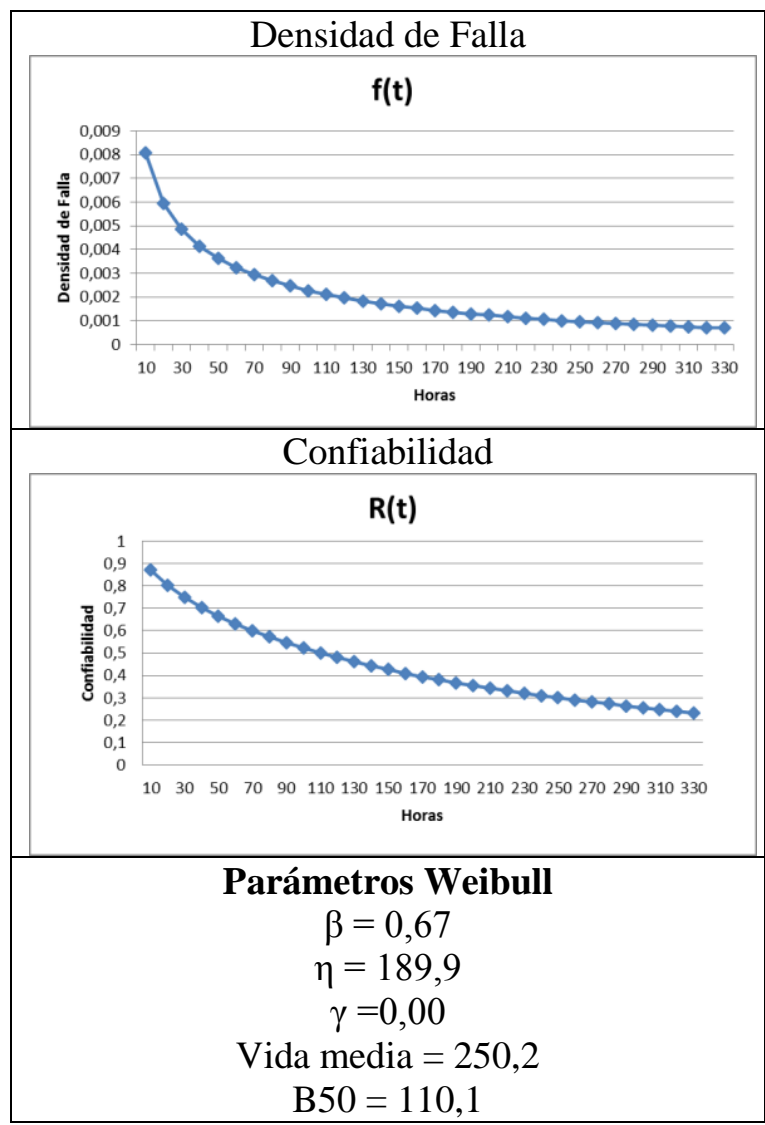

\subsection{Análisis de funcionalidad e identificación de los modos de falla}

Desde el punto de vista de la función que cumplen cada uno los seis equipos principales, son solamente cuatro tipos de equipos distintos: enfriadores (1 y 2); tornillos de transporte (13 y 14); la rastra y el transportador neumático.

\section{Enfriadores de Calcina.}

Son los equipos encargados de bajar la temperatura de la calcina, desde $550^{\circ} \mathrm{C}$ en la entrada, hasta $80^{\circ} \mathrm{C}$ en la salida. Esto ocurre a una tasa de 40 ton/hr., con una velocidad de rotación de 4 RPM. Al realizar el análisis de modos de falla, se encuentran los fallos funcionales principales: enfriador no gira; enfriador no enfría; alta temperatura de salida agua de enfriamiento (mayor a $60{ }^{\circ} \mathrm{C}$ ); pérdida de agua de refrigeración; fuga de calcina hacia el exterior.

\section{Rastra 3.}

Equipo encargado de transportar de manera continua calcina a $80^{\circ} \mathrm{C}$ desde la rastra 9 y rastra 10 hacia el chute de descarga y el harnero vibratorio, a razón de 100 Ton/hr, Los fallos funcionales principales son: rastra no transporta calcina; fuga de calcina hacia el exterior.

\section{Tornillos.}

Cumplen la función principal de transportar de manera continua calcina a $500^{\circ} \mathrm{C}$ desde el ciclón primario hacia el chute de descarga y hacia el enfriador, a razón de 50 Ton/hr. Su función debe ser cumplida, sin que haya pérdida de calcina, sin pérdida de agua de refrigeración, y sin humedad en calcina. Los componentes críticos de mayor tasa de falla son packing de estopa, cadena motriz y descanso motriz, los cuales son reemplazados en la mayoría de los casos, cuando presentan falla. Las principales fallas funcionales detectadas para el tornillo de transporte son: tornillo no transporta calcina; pérdida de agua de refrigeración; fuga de calcina hacia el exterior.

\section{Transporte Neumático.}

Transporta de manera continua calcina a $80^{\circ} \mathrm{C}$ desde la rastra 3 hacia las tolvas de despacho de 
200

calcina, a razón de 100 Ton/hr. Su principal falla funcional es que no haya transporte de calcina.

\subsection{FMECA-RCM aplicado a los enfriadores}

Para el análisis que se describe a continuación, se considera como modelo el caso del enfriador de calcina No.1, dada su alta criticidad por el elevado número de horas de detención que ha presentado comparado con los demás equipos principales. Esto, a pesar de que el análisis se realiza de manera completa para todos los equipos principales de la planta de tostación (Cordero y Estupiñan, 2018).

La estrategia de mantenimiento para los enfriadores está basada en la inspección de componentes críticos. Usualmente las inspecciones consideran rutinas de medición de vibraciones (elementos rodantes, rodillos y motor eléctrico); y rutas de lubricación (rodillos, motor, reductor y cadena de transmisión). Complementariamente, se llevan a cabo inspecciones visuales, monitoreo de comportamiento de corriente eléctrica y nivel del estanque de agua desmineralizada. Generalmente, cuando existen componentes críticos con algún tipo de fallo que impide la operación óptima del enfriador, se realiza una detención no programada, con el fin de dar solución a la falla y seguir operando. Según los datos históricos tomados para el estudio, los componentes con mayor tasa de falla son los "cassettes". En los últimos 12 meses, se han cambiado 24 cassettes en mantenciones programadas y 18 cassettes en mantenciones no programadas. Además, debido a fallos inesperados, se han debido cambiar otros componentes principales tales como los sellos.

Realizando el análisis mediante la metodología FMECA, la cual permite reconocer la naturaleza de las fallas, las necesidades del mantenimiento y las áreas de mejora potenciales; y complementando el análisis mediante el árbol de decisión (metodología RCM), se puede obtener una lista completa priorizada de las actividades de mantenimiento y de los repuestos críticos (Cordero y Estupiñan, 2018). Como resultado de este análisis, se puede ver la propuesta de optimización del plan de mantenimiento para los modos de falla críticos del enfriador, en anexo 1.

\section{Análisis de Sensibilidad}

Se realizó un análisis de sensibilidad, con el fin de estimar el incremento de la confiabilidad y la reducción de los costos por pérdidas de producción y gastos de mantenimiento, con la propuesta de plan de mantenimiento optimizada para el enfriador de calcina 1. Para ello, se considera una producción de calcina de la planta de 69 Ton/hr., con un costo aproximado de 1200 USD/Ton. Además, respecto de los gastos de mantenimiento, sólo se consideran aquellos debidos a sobre-consumo de repuestos, ya que no hay sobre costo por personal dado los turnos fijos día y noche con que ya se cuenta en la planta. Por lo tanto, el gasto causado por pérdidas de producción según el plan actual (considerando: 240 horas detención, a 1200 US/Ton, 69 Ton/hr.), asciende a la suma de MU\$19830, adicional a un sobreconsumo de repuestos por la suma MU\$265; obteniendo un total de pérdidas por MU\$ 20095 dólares.Por otra parte, si se utiliza el plan de mantenimiento optimizado, el recambio de componentes debiese disminuir la ocurrencia de fallas imprevistas. Si se estima que el número de detenciones imprevistas, que generan pérdidas de producción, disminuye de 35 a 13 eventos, con un total de 36.3 horas de detención; el gasto asociado a pérdidas por producción sumado al gasto por sobreconsumo de repuestos (aprox. U\$1000, por concepto de cambio de sellos de entrada y salida); asciende a la suma de MU\$3006. Los parámetros de confiabilidad, tomando en cuenta la nueva propuesta de plan de mantenimiento para el enfriador 1, y considerando la disminución de las detenciones imprevistas; se muestran en la tabla 4. 
201

Tabla 4. Curvas de densidad de falla y confiabilidad, para el Enfriador 1 (con plan propuesto)

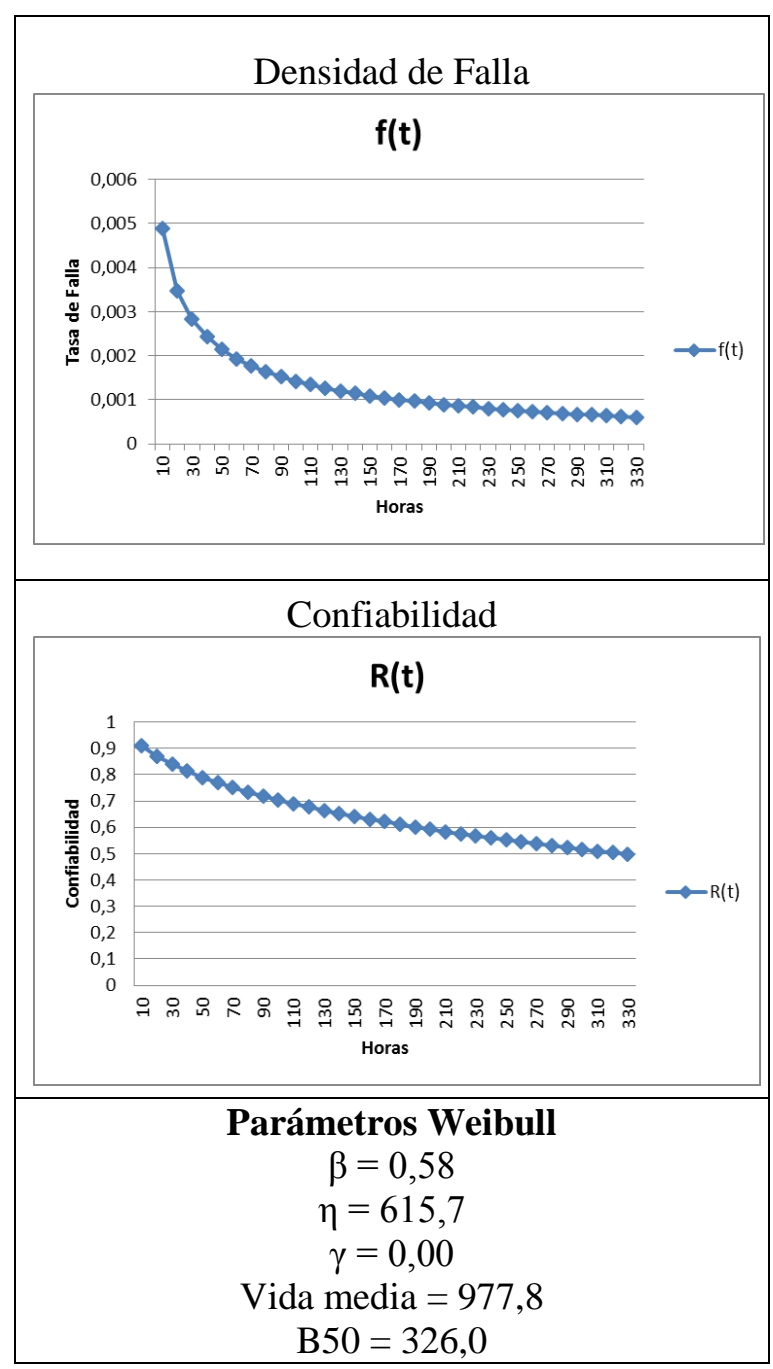

Como se puede observar de los gráficos y resultados mostrados en la tabla 4 , comprándolos con la tabla 3, se aprecia una mejora en los valores de vida media y tiempo en probabilidad de falla al $50 \%$ que suben de 250 a 977,8 horas para el caso de vida media, y de 110 a 326 horas en el caso del B50. También se aprecia mejora en el valor de $\eta$, parámetro que concentra el $62,3 \%$ de la fallas, y que cambia de 189 a un nuevo valor de 615 horas. En resumen se puede apreciar que con el plan propuesto, la confiabilidad del equipo aumenta, como también, hay una significativa disminución de las pérdidas o gastos relacionados con mantenimiento, por un monto aproximado de MU\$17089. Sí se aplica la metodología para los demás equipos principales de la planta de tostación, y se comparan las pérdidas actuales con las proyectadas considerando los planes de mantenimiento optimizados, se obtiene una reducción de gastos por mantenimiento principalmente, debido a imprevistos $\mathrm{y}$ sobreconsumo de repuestos, por un monto cercano a los MU\$30000; y logrando un gasto optimizado por concepto de mantenimiento, por un monto de MU\$4895, considerando todos los equipos principales de la planta de tostación. Respecto de la disponibilidad, se obtiene una mejora importante, pasando del $83,25 \%$, al $87,28 \%$, con el plan propuesto.

\section{Conclusiones}

Debido a la naturaleza de la operación de la planta de tostación, se ha podido constatar que la metodología FMECA - RCM, permite evaluar los equipos principales, de tal manera de poder contar con una propuesta optimizada de plan de mantenimiento, para cada uno de los equipos críticos de la planta. De esta manera, se busca disminuir las detenciones imprevistas, las cuales producen daño progresivo a la integridad del lecho del horno de tostación.

Un enfoque de mantenimiento basado en confiabilidad, permite además acortar el tiempo de las detenciones, y disminuir las pérdidas asociadas a las mismas. Se pudo constatar mediante el estudio, que las pérdidas asociadas a costos de producción y sobreconsumo de repuestos por fallas imprevistas de equipos son muy cuantiosas alcanzando el valor cercano a los MU\$35000. Lo anterior, considerando solamente seis equipos, que abarcan el $95 \%$ de las detenciones imprevistas durante un año de estudio, en el Complejo de Tostación de la División Ministro Hales de Codelco. 
Luego de realizar el análisis de sensibilidad y utilizando la metodología y planes propuestos se estima alcanzar una reducción de costos de MU\$30000, que están asociados principalmente a pérdidas de producción y sobre-consumo de repuestos.

\section{Referencias Bibliogràfícas}

Luthra, P. (1991). FMECA: an integrated approach. Annual Reliability and Maintainability Symposium. Proceedings, Orlando, FL, pp. 235-241.

Moubray, j. (2001). RCM Reliability Centred Maintenance, Industrial Press Inc.

Quintana, A.E., Pisani, M.V., Casal, R.N. (2015). Desempeño de cartas de control estadístico con limites bilaterales de probabilidad para monitorear procesos Weibull en mantenimiento. Ingeniería, Investigación y Tecnología, January-March, Vol.16 (1), pp.143-156.

International Standard Organization. (2011). ISO 17359: Condition monitoring and diagnostics of machines -- General guidelines.

Waghmode, L.Y. and Sahasrabudhe, A.D. (2012). Modelling maintenance and repair costs using stochastic point processes for life cycle costing of repairable systems. International Journal of Computer Integrated Manufacturing. Apr/May, Vol. 25, Issue 4/5, pp. 353-367.

Cordero, O. and Estupiñan, E. (2018). Propuesta de optimización del mantenimiento de planta minera de cobre Ministro Hales, mediante análisis de confiabilidad, utilizando la metodología FMECA. Revista Investigación y Desarrollo, Vol. 18 (1), pp. 129-142. 


\section{Anexo 1.}

Plan de mantenimiento para los modos de falla críticos de los enfriadores

\begin{tabular}{|c|c|c|c|c|c|c|c|}
\hline $\begin{array}{c}\text { MODO DE } \\
\text { FALLA }\end{array}$ & $\begin{array}{l}\text { Sustitución } \\
\text { Preventiva }\end{array}$ & $\begin{array}{l}\text { Reacondicionamiento } \\
\text { Ciclíco Preventivo }\end{array}$ & $\begin{array}{l}\text { Inspeccion } \\
\text { MONCON }\end{array}$ & $\begin{array}{l}\text { Reparación a la } \\
\text { condición }\end{array}$ & $\begin{array}{c}\text { Reparación } \\
\text { a la falla }\end{array}$ & $\begin{array}{l}\text { Repuesto } \\
\text { Critico }\end{array}$ & Cantidad \\
\hline $\begin{array}{l}\text { CORTE CADENA } \\
\text { TRANSMISIÓN }\end{array}$ & 1 vez al año & No aplica & $\begin{array}{l}1 \text { vez por } \\
\text { semana }\end{array}$ & $\begin{array}{c}\text { Si existen saltos de } \\
\text { cadena }\end{array}$ & No aplica & $\begin{array}{c}\text { Cadena } \\
\text { transmisión }\end{array}$ & 1 \\
\hline $\begin{array}{l}\text { FALLA MACHON } \\
\text { ACOPLE }\end{array}$ & 1 vez al año & No aplica & $\begin{array}{l}1 \text { vez por } \\
\text { semana }\end{array}$ & $\begin{array}{c}\text { Si hay aumento en } \\
\text { vibración de } \\
\text { sistema }\end{array}$ & No aplica & $\begin{array}{c}\text { Machon de } \\
\text { Acoplamiento }\end{array}$ & 1 \\
\hline FALLA REDUCTOR & $\begin{array}{c}1 \text { vez cada } 2 \\
\text { años }\end{array}$ & No aplica & $\begin{array}{l}1 \text { vez por } \\
\text { semana }\end{array}$ & $\begin{array}{c}\text { Si hay aumento en } \\
\text { vibración de } \\
\text { sistema }\end{array}$ & No aplica & Reductor & 1 \\
\hline $\begin{array}{l}\text { FALLA EN MOTOR } \\
\text { ELÉCTRICO }\end{array}$ & 1 vez al año & No aplica & $\begin{array}{l}1 \text { vez por } \\
\text { semana }\end{array}$ & \begin{tabular}{|c} 
Si hay aumento en \\
vibración de \\
sistema/Temperat \\
ura/Corriente
\end{tabular} & No aplica & Motor Eléctrico & 1 \\
\hline $\begin{array}{l}\text { FALLA SPROCKET } \\
\text { MOTRIZ O TENSOR }\end{array}$ & \begin{tabular}{|c|}
1 vez cada 2 \\
años \\
cambiar \\
sprocket \\
motriz \\
\end{tabular} & $\begin{array}{l}\text { Sprocket tensor } \\
\text { repara } 1 \text { vez al año } \\
\text { buje }\end{array}$ & $\begin{array}{l}1 \text { vez por } \\
\text { semana }\end{array}$ & $\begin{array}{c}\text { Si existe soltura en } \\
\text { ajuste }\end{array}$ & No aplica & $\begin{array}{c}\text { Sprocket } \\
\text { Motriz/Tensor }\end{array}$ & 1 \\
\hline RODILLOS TRANCADOS & $\begin{array}{c}1 \text { vez cada } 2 \\
\text { años }\end{array}$ & No aplica & $\begin{array}{l}1 \text { vez por } \\
\text { semana }\end{array}$ & $\begin{array}{c}\text { Si existe } \\
\text { soltura/Temperatur } \\
\text { a/vibración/Desga } \\
\text { ste }\end{array}$ & No aplica & $\begin{array}{l}\text { Rodillos } \\
\text { Soporte y } \\
\text { Axiales }\end{array}$ & 4 \\
\hline CASSETTE OBSTRUIDO & No Aplica & No aplica & $\begin{array}{l}\text { Todos los } \\
\text { dias }\end{array}$ & $\begin{array}{c}\text { Si existe alta } \\
\text { temperatura en } \\
\text { cabezal de } \\
\text { cassette y salida } \\
\text { de agua } \\
\end{array}$ & No aplica & Cassette & - \\
\hline $\begin{array}{l}\text { BAJO CAUDAL AGUA } \\
\text { REFRIGERACIÓN POR } \\
\text { ROTURA INTERNA }\end{array}$ & 1 vez al año & No aplica & $\begin{array}{l}\text { Todos los } \\
\text { dias }\end{array}$ & $\begin{array}{l}\text { Si existe Fuga de } \\
\text { Agua }\end{array}$ & No aplica & \begin{tabular}{|c|c|}
$\begin{array}{c}\text { Flexibles de } \\
\text { entrada/salida } \\
\text { /manto }\end{array}$ \\
\end{tabular} & 72 \\
\hline $\begin{array}{c}\text { BAJO CAUDAL AGUA } \\
\text { REFRIGERACIÓN POR } \\
\text { BAJA EFICIENCIA } \\
\text { BOMBAS }\end{array}$ & 1 vez al año & No aplica & $\begin{array}{l}1 \text { vez por } \\
\text { semana }\end{array}$ & \begin{tabular}{|c} 
Si hay aumento en \\
vibración de \\
sistema/Baja \\
eficiencia
\end{tabular} & No aplica & $\begin{array}{c}\text { Bombas de } \\
\text { agua } \\
\text { desmineralizad } \\
\text { a }\end{array}$ & 1 \\
\hline $\begin{array}{c}\text { OBSTRUCCIÓN } \\
\text { INTERCAMBIADOR DE } \\
\end{array}$ & No aplica & Cada 6 meses & $\begin{array}{l}1 \text { vez por } \\
\text { semana }\end{array}$ & Por baja Eficiencia & No aplica & \begin{tabular}{|c|} 
Placas \\
Intercambiador
\end{tabular} & 1 \\
\hline $\begin{array}{l}\text { ROTURA INTERNA DE } \\
\text { CASSETTE }\end{array}$ & $\begin{array}{l}\text { Cada } 4 \\
\text { Meses }\end{array}$ & No aplica & $\begin{array}{l}\text { Todos los } \\
\text { dias }\end{array}$ & $\begin{array}{c}\text { Perdida brusca de } \\
\text { agua } \\
\text { desmineralziada/ } \\
\text { Humedad en la } \\
\text { calcina }\end{array}$ & No aplica & Cassette & 18 \\
\hline $\begin{array}{l}\text { ROTURA INTERNA } \\
\text { PIPING DE AGUA } \\
\text { REFRIGERACIÓN }\end{array}$ & 1 vez al año & No aplica & $\begin{array}{l}\text { Todos los } \\
\text { dias }\end{array}$ & \begin{tabular}{|c|} 
Perdida brusca de \\
agua \\
desmineralziada/ \\
Humedad en la \\
calcina \\
\end{tabular} & No aplica & Manifold Agua & 1 \\
\hline $\begin{array}{l}\text { ROTURA SELLOS } \\
\text { ENTRADA/SALIDA }\end{array}$ & cada 15 dias & No aplica & $\begin{array}{l}\text { Todos los } \\
\text { dias }\end{array}$ & $\begin{array}{c}\text { Si hay Polución de } \\
\text { calcina }\end{array}$ & No aplica & $\begin{array}{c}\text { Sellos polvo } \\
\text { entrada /salida }\end{array}$ & 24 \\
\hline ROTURA SELLO TROMEL & 1 vez al año & No aplica & $\begin{array}{c}\text { Todos los } \\
\text { dias }\end{array}$ & $\begin{array}{c}\begin{array}{c}\text { Si hay Polución de } \\
\text { calcina }\end{array} \\
\end{array}$ & No aplica & $\begin{array}{c}\text { Sello Grafito } \\
\text { Tromel }\end{array}$ & 1 \\
\hline $\begin{array}{c}\text { ROTURA MANTO } \\
\text { ENDRIADOR }\end{array}$ & No aplica & No aplica & $\begin{array}{c}\text { Todos los } \\
\text { dias }\end{array}$ & $\begin{array}{c}\begin{array}{c}\text { Si hay Polución de } \\
\text { calcina }\end{array} \\
\end{array}$ & No aplica & No aplica & No aplica \\
\hline $\begin{array}{l}\text { CANDADOS SUELTOS } \\
\text { CASSETTE }\end{array}$ & No aplica & cada 15 dias & $\begin{array}{l}\text { Todos los } \\
\text { dias }\end{array}$ & $\begin{array}{c}\text { Si hay Polución de } \\
\text { calcina }\end{array}$ & No aplica & Candados & 108 \\
\hline
\end{tabular}


*Para citar este artículo: Estupiñan E.; Oscar Cordero O.Using FMECA - RCM methodology as a tool for the optimization of the maintenance strategy in a copper roasting plant. Revista Bistua. 2019 17(1):196-204

+ Autor para el envió de correspondencia y la solicitud de las separatas: Estupiñan E Departamento de Ingeniería Mecánica, Universidad de Tarapacá (Arica, Chile). eestupin@uta.cl

Recibido: Marzo 22 de 2018

Aceptado: Agosto 17 de 2018 